\title{
RELAP5-3D Developer Guidelines and Programming Practices
}

George L. Mesina

June 2013

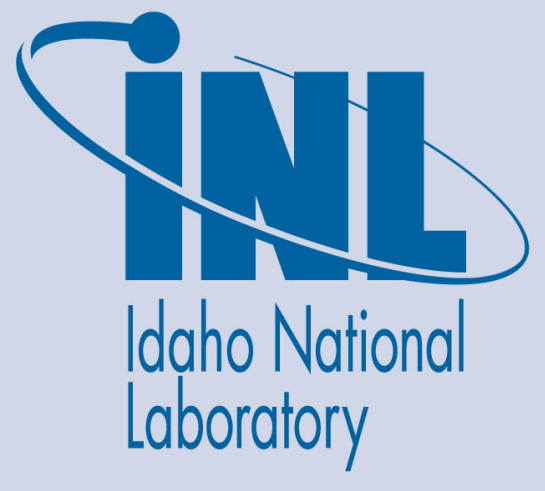

The INL is a U.S. Department of Energy National Laboratory operated by Battelle Energy Alliance 
INL/EXT-13-29228

Rev. 1

\title{
RELAP5-3D Developer Guidelines and Programming Practices
}

\author{
George L. Mesina
}

June 2013

\author{
Idaho National Laboratory \\ Thermal Science and Safety Analysis \\ Idaho Falls, Idaho 83415
}

http://www.inl.gov

\author{
Prepared for the \\ Office of Naval Reactors \\ and for the \\ U.S. Department of Energy \\ Under DOE Idaho Operations Office \\ Contract DE-AC07-05ID14517
}


INL/EXT-13-29228

Revision 1

\section{RELAP5-3D Developer Guidelines and Programming Practices}

Dr. George L Mesina

June 26, 2013

The INL is a U.S. Department of Energy National Laboratory operated by Battelle Energy Alliance 
RELAP5-3D Developer Guidelines and Programming Practices

Dr. George L Mesina

June, 2013

\author{
Idaho National Laboratory \\ Thermal Science and Safety Analysis \\ Idaho Falls, Idaho 83415 \\ http://www.inl.gov
}

\author{
Prepared for the \\ U.S. Department of Energy \\ Office of Naval Reactors \\ Under DOE Idaho Operations Office \\ Contract DE-AC07-05ID14517
}



RELAP5-3D Developer Guidelines and Programming Practices

\title{
RELAP5-3D Developer Guidelines and Programming Practices
}

\author{
INL/EXT-13-29228
}

June, 2013

Approved by:

Dr. George Mesina

Author

Date

Nolan Anderson

Date

Technical Reviewer

Dr. James Wolf

Date

Project Manager

George Griffith

Date

Department Manager 


\section{RELAP5-3D Developer Guidelines and Programming Practices}

\section{EXECUTIVE SUMMARY}

Our ultimate goal is to create and maintain RELAP5-3D as the best software tool available to analyze nuclear power plants. This begins with writing excellent programming and requires thorough testing. This document covers development of RELAP5-3D software, the behavior of the RELAP5-3D program that must be maintained, and code testing.

RELAP5-3D must perform in a manner consistent with previous code versions with backward compatibility for the sake of the users. Thus file operations, code termination, input and output must remain consistent in form and content while adding appropriate new files, input and output as new features are developed.

As computer hardware, operating systems, and other software change, RELAP5-3D must adapt and maintain performance. The code must be thoroughly tested to ensure that it continues to perform robustly on the supported platforms.

The coding must be written in a consistent manner that makes the program easy to read to reduce the time and cost of development, maintenance and error resolution.

The programming guidelines presented her are intended to institutionalize a consistent way of writing FORTRAN code for the RELAP5-3D computer program that will minimize errors and rework. A common format and organization of program units creates a unifying look and feel to the code. This in turn increases readability and reduces time required for maintenance, development and debugging. It also aids new programmers in reading and understanding the program. Therefore, when undertaking development of the RELAP5-3D computer program, the programmer must write computer code that follows these guidelines.

This set of programming guidelines creates a framework of good programming practices, such as initialization, structured programming, and vector-friendly coding. It sets out formatting rules for lines of code, such as indentation, capitalization, spacing, etc. It creates limits on program units, such as subprograms, functions, and modules. It establishes documentation guidance on internal comments.

The guidelines apply to both existing and new subprograms. They are written for both FORTRAN 77 and FORTRAN 95. The guidelines are not so rigorous as to inhibit a programmer's unique style, but do restrict the variations in acceptable coding to create sufficient commonality that new readers will find the coding in each new subroutine familiar.

It is recognized that this is a "living" document and must be updated as languages, compilers, and computer hardware and software evolve. 


\section{RELAP5-3D Developer Guidelines and Programming Practices}

\section{CONTENTS}

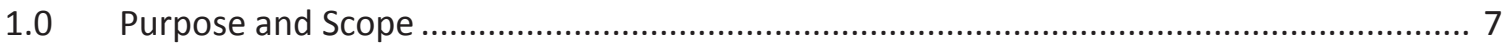

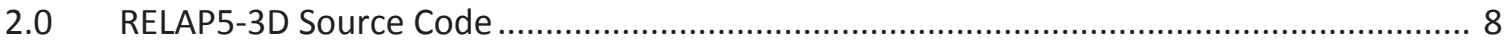

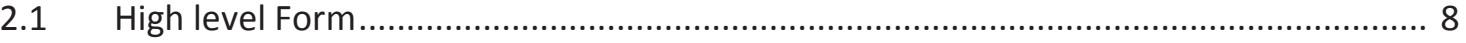

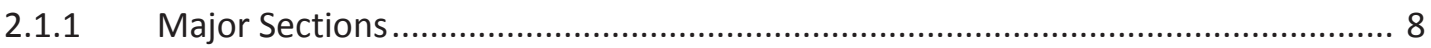

2.1.2 Structured Programming Paradigm....................................................................... 8

2.2 Formatting Statements of Source Code .................................................................. 10

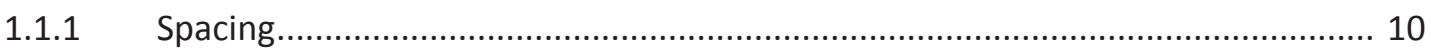

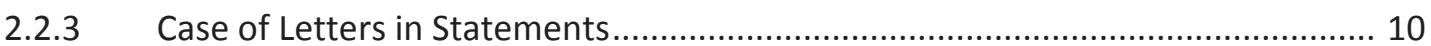

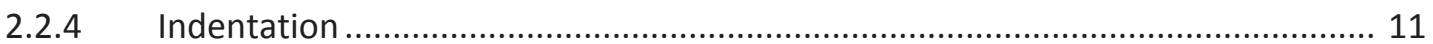

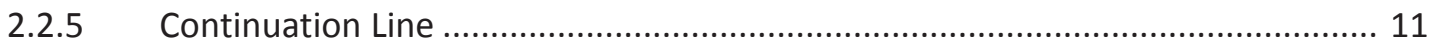

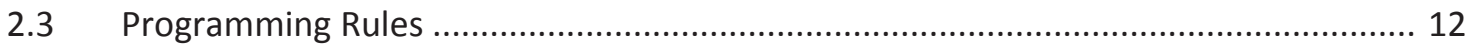

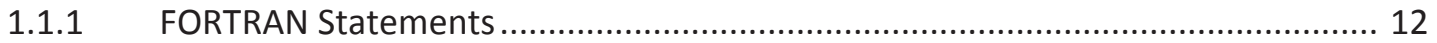

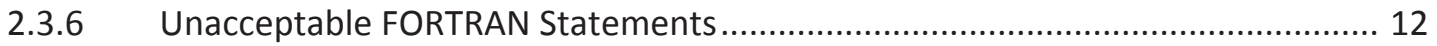

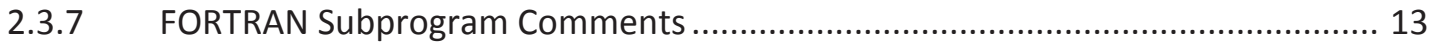

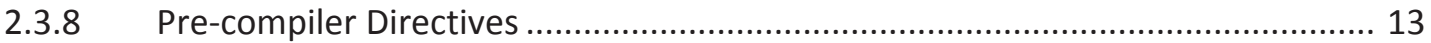

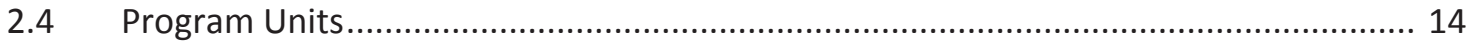

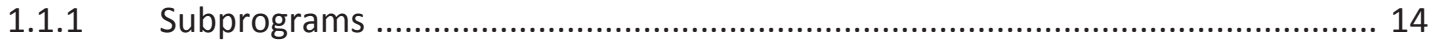

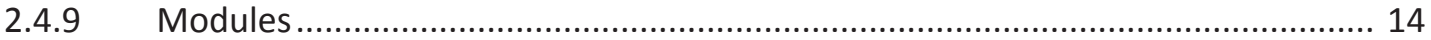

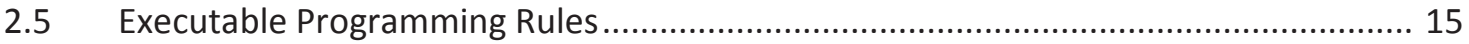

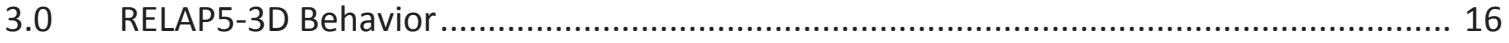

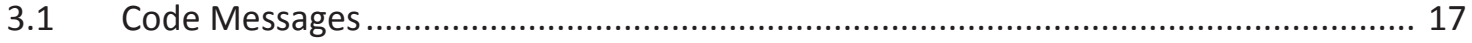

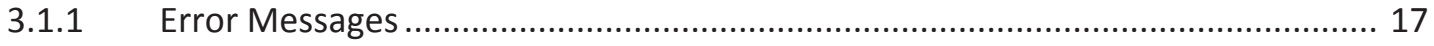

3.1.2 Warning Message Formats ............................................................................. 17

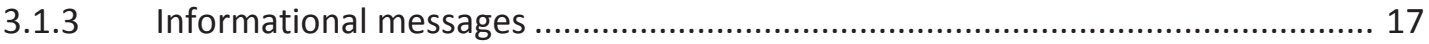

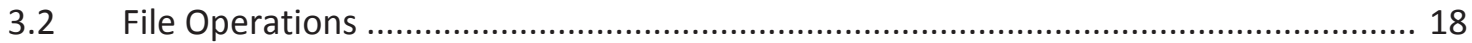

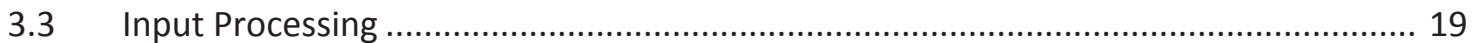

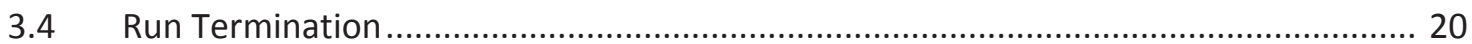

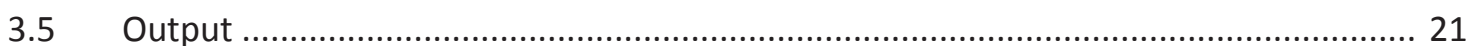

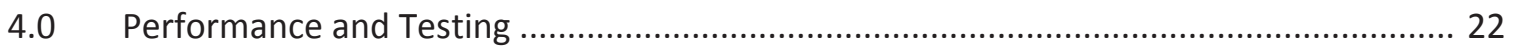

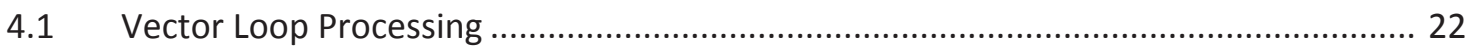

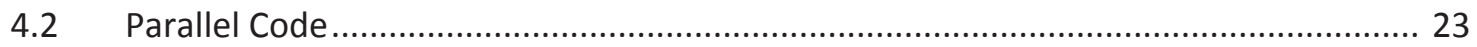

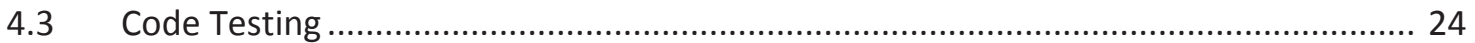




\section{RELAP5-3D Developer Guidelines and Page 6 Programming Practices

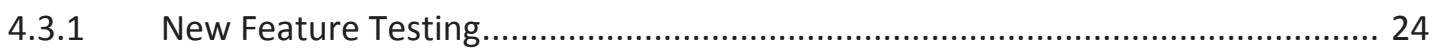

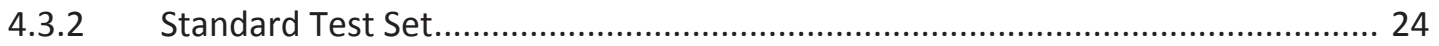

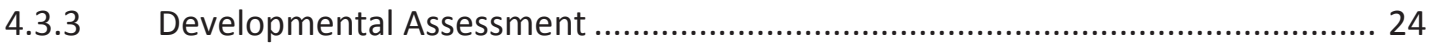

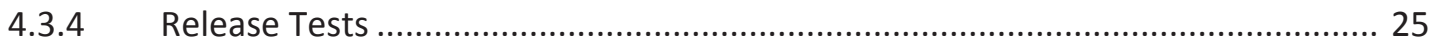

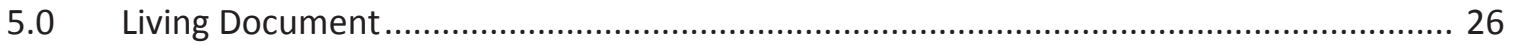




\section{RELAP5-3D Developer Guidelines and Programming Practices}

\subsection{Purpose and Scope}

Our ultimate goal is to create and maintain the best software tool available to analyze nuclear power plants. This is a lofty ideal. It begins with having excellent programming and requires thorough testing.

In Section 2, RELAP5-3D Source Code is addressed. Developers are responsible for writing code in a manner consistent with the guidelines. Staying within the guidelines makes the program easy to read to reduce the time and cost of development, maintenance and error resolution.

In Section 3, RELAP5-3D behavior is described. RELAP5-3D must perform in a manner consistent with previous code versions for the sake of the users. Thus file operations, code termination, input and output must remain consistent in form and content while adding appropriate new files, input and output as new features are developed. Backward compatibility is very important for input processing for many users. Developers are responsible for not changing code behavior by the updates they submit and for producing the same consistent for new code features, error fixes, and maintenance.

In Section 4, testing is explained. Developers are responsible for creating input that tests their coding updates, testing their code developments, and providing a test set to the code version maker to include in the RELAP5-3D test sets. As computer hardware, operating systems, and other software change, RELAP5-3D must adapt and maintain performance. The code must be thoroughly tested to ensure that it continues to perform robustly on the supported platforms. 


\section{RELAP5-3D Developer Guidelines and Programming Practices}

\subsection{RELAP5-3D Source Code}

\subsection{High level Form}

RELAP5-3D program units should have the professional look of other library software such as IMSL or those published in books like Numerical Recipes. Moreover, subprograms should be written to produce the correct result while running as quickly as possible. The organization of program units into major parts is explained in 2.1.1. The concept of structured programming paradigm is explained in 2.1.2.

\subsubsection{Major Sections}

Modules, subroutines, and functions have major sections that occur in a particular order to create uniformity and ease in locating information.

For a FORTRAN 95 module the major sections are

(1) Module header statement

(2) Module identification comments after module statement

(3) PART 1 - Declaration section

(4) PART 2 - Documentation section

(5) PART 3 - Internal subprograms

The major elements of a subprogram unit (whether independent or internal) occur in the following order:

(1) Subprogram header statement

(2) Subprogram identification comments after subprogram statement

(3) Declaration section organized according to Section 2.2

(4) Data dictionary that defines all local variables and call arguments

(5) An "Executable Code" comment that separates the non-executable top of the program unit from the executable section

(6) Outline style comments throughout the body of the executable code

(7) Body of the program unit written in uniform programming style in accordance with the rest of this guideline

\subsubsection{Structured Programming Paradigm}

Structured programming is considered the most strongly modular form of coding, even stronger than object-oriented programming. It makes a code very readable.

A structured program unit is written as a sequence of blocks-of-code and sub-blocks-of-code. A block-of-code in FORTRAN is one of the following programming constructs: do-loop, if-then-else, case, and non-branching statement (assignment, I/O, etc.).

The following rules are strictly enforced to have structured programming:

(1) No jumps (via go to statements) into a block or sub-block from outside it

(2) No jumps from inside a block or sub-block to outside it

(a) Jumps may only terminate inside the block or at its bottom end

(3) No backwards jumps except via a do-loop construct. 


\section{RELAP5-3D Developer Guidelines and Page 9 Programming Practices

(4) Blocks may not co-terminate. Thus for example, an outer and inner do-loop may not end on the same statement. 


\section{RELAP5-3D Developer Guidelines and Programming Practices}

\subsection{Formatting Statements of Source Code}

The following is a set of programming requirements and conventions for formatting lines of code. This is intended to make the code more readable and easier to maintain. There are two reasons for doing this. The first is to establish a uniform coding style that is flexible enough to allow some individuality, yet allow everyone familiar with the style to recognize other people's programming quickly and easily. The second is to allow new developers with a programming background in $\mathrm{C}++$, Java or similar languages to quickly become productive with RELAP5-3D by employing modern programming constructs and styles with which they've become familiar.

\subsubsection{Spacing}

1) Each FORTRAN keyword should have a space on either side of it. Keywords that have an argument list should have a space after the closing parenthesis.

2) The elements of a keyword's argument should have a space after each comma, though this is optional.

3) Lists of variables should have a single space after each comma. On I/O statements the space after comma is optional though preferred.

4) Assignment and do statement equal signs should have a space on either side.

5) Spaces should be placed on either side of + and - arithmetic operator. Exception: omit spaces around + and - inside index calculations.

6) Do not put spaces around $* *, *$ and / operators.

7) Spaces around logical operators are optional.

For example, replace

$$
\begin{aligned}
& \text { do } \quad i 1=1, \text { nvar, } 2 \\
& i=i 1+j 1^{*} \text { ncolumns }+k 1 * n p l \text { anes } \\
& \text { read (unit, format, end=100, err=200) a,b, c ( } i+1)
\end{aligned}
$$

by

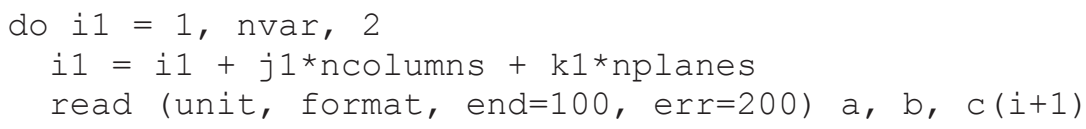

\subsubsection{Case of Letters in Statements}

Upper and lower case letters can be used to aid readability. Just like in a book, most text should be in lower case. Use of upper case can improve readability if used in a predictable and uniform manner.

All FORTRAN 77 executable source code should be in lower case. This does not apply to comments.

FORTRAN 95 source should either use lower case or camelback notation for variable names. Camelback puts a capital at the start of each word within a variable name. For example:

- auxfilename is lower case

- auxFileName is camelback.

FORTRAN 95 keywords may use all capital letters; this is optional. 


\section{RELAP5-3D Developer Guidelines and Programming Practices}

\subsubsection{Indentation}

In some RELAP5-3D program units, indentation can be very deep, exceeding 10 levels and even reaching 15 in places. To prevent long and deeply indented FORTRAN 77 statements having too many continuation lines, the standard of two space indentation was created. For uniformity, this is applied to FORTRAN 95 coding also.

Initial indentation and length of lines in a statement are also made uniform. The following rules apply:

1) Indentation is two spaces for each level of indentation.

2) Do not use tabs for indentation in FORTRAN coding.

3) Leftmost coding in FORTRAN 77 coding is column 8.

4) Leftmost coding in FORTRAN 95 goes in column 2.

5) Comments begin in column 1 in both FORTRAN 77 an 95 coding; however, FORTRAN 95 coding indent comments if it improves readability.

\subsubsection{Continuation Line}

In FORTRAN 77 statements, use the " $\&$ " ampersand symbol in column 6.

In FORTRAN 95 statements, use the "\&" ampersand symbol at the end of the line at least 10 spaces to the right of the last non-blank unless it is arranged in the same column as an ampersand in the line immediately above or below it. Lining up continuation marks improves readability. 


\section{RELAP5-3D Developer Guidelines and Programming Practices}

\subsection{Programming Rules}

The following is a set of programming requirements and conventions. Much of this is intended to make the code more readable and easier to maintain. There is need to eliminate certain archaic programming practices that are hard to read, time-consuming to decipher, prone to difficulty debugging, and/or have been listed as obsolete or deprecated in the FORTRAN 95 ANSI standard.

\subsubsection{FORTRAN Statements}

It is allowable to use every type of non-obsolesced statement in the ANSI FORTRAN 77 and FORTRAN 95 standard in the files or the respective types.

1. Use "implicit none" in every program unit, module and subprogram.

2. Use the module named intrtype in all program units.

3. Declare every variable in a program unit.

4. Alphabetize declaration lists for each data type.

5. The order of declaration statements is:

a. Derived type creation, derived type instances, arrays, scalars

b. Declare FORTRAN basic types in this order:
i. Integer
ii. Real
iii. Logical
iv. Character

6. Initialize all variables.

7. Nullify all pointers

a. When they are created, if that does not ruin the algorithm.

8. Deallocate all subtypes of derived type quantities before deallocating the instance of a derived type.

a. This eliminates memory leaks.

9. Use format statements unless the format can fit on one line within the confines of the argument list of the I/O statement.

\subsubsection{Unacceptable FORTRAN Statements}

Many features have become obsolescent in later FORTRAN standards. Though these statements are still legal FORTRAN, they should not be used in any new coding. Moreover, there are many perfectly legitimate FORTRAN constructs and statements that should also not be used in any new coding. These items have caused much difficulty with debugging and maintenance and give new programmers great trouble.

1. Do not introduce any NEW bit-packing.

2. Do not introduce any new assigned go to or computed go to statements.

3. Do not use backward go to statements. See the code architect if you cannot find a way to avoid introducing one.

4. Replacement: FORTRAN 90 "case" statement or FORTRAN 77 multiple elseif conditional statement (if/then/else/else if/ ... /else if/end if).

5. Do not introduce new equivalence or common statements. 


\section{RELAP5-3D Developer Guidelines and Programming Practices}

6. Never introduce machine-dependent compiler extensions. Stick with the ANSI FORTRAN 95 standard.

7. Do not exceed array index bounds. Checking array bounds with compiler options is encouraged.

8. Avoid placing multiple return statements in a program unit.

\subsubsection{FORTRAN Subprogram Comments}

Internal documentation is important to identify the program unit, author, creation dates as well as give its purpose, provide a dictionary of its variables, and outline its operations. This section formalizes the kinds and locations of comments in program units. It is reasonable to have between $25 \%$ and $50 \%$ of the lines of code be comments.

1. Subroutine identification documentation includes
a. subprogram purpose
b. creation/update date
c. cognizant engineer

2. Data Dictionary should occur after the declarations

a. Alphabetize the dictionary variables

b. Use Capital letters in the definition to show how the variable name relates.

c. Line up the definitions to all start in the same column.

3. Comments within the body of the subprogram should be outline style.

a. Each major section should receive a section title and short description.

b. Each important minor section should receive a subsection title.

c. Each heading should be numbered outline style (publication style)

Example: a major section heading might be "1.0 Input."

Example: a minor section heading might be "1.1 Argument checking."

4. Blank Comment Line

a. A blank comment may precede a significant comment line for readability.

5. Spacing

a. Each comment should have a ! in column 1 and the text should begin in column 4 .

b. Begin each sentence/phrase with a capital letter and end with a period.

\subsubsection{Pre-compiler Directives}

1) Do not introduce any pre-compiler directives that have a line count. For example: \#if def,mystuff,1

$$
\text { Write }(*, *) \text { diagnostic }
$$

This should be coded as:

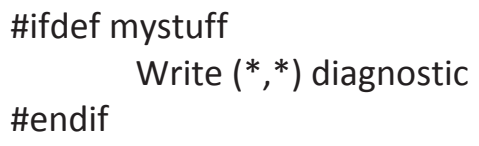

2) Do not begin pre-compiler directives names with a number.

3) Do not name pre-compiler directives with a common word as those words can be removed throughout the code by the pre-compiler. 


\section{RELAP5-3D Developer Guidelines and Programming Practices}

\subsection{Program Units}

\subsubsection{Subprograms}

Subprograms should not have so many lines of coding that they become hard to read, understand, debug, develop, and maintain.

New subprogram should not exceed 200 lines. If one becomes too long in its main routine, break out subsections to create internal subprograms or new external subprograms.

Internal subprograms should not exceed 200 lines, and with rare exceptions, should be shorter than the main subprogram to which they are internal. Internal subprograms can be further divided into smaller internal subroutines, and one internal routine can call another.

\subsubsection{Modules}

Use statements should make use of the "only" construct. Limit the items "used" in a module or subprogram to just those required. For example, if variables $A A$ and $B B$ are the only items from module $A B C$, then rather than

use abc

program

use abc, only: aa, bb

Beware of using modules inside other modules. The only exception is intrtype which should be used in all RELAP5-3D modules.

Using one module inside another creates a dependency and forces order precedence on compilation. This means the used module must be compiled before its user-module. This can create recursion (E.G. Module A uses Module B which uses Module C which uses Module A) and makes compilation impossible until it is resolved.

In general, it is best combine modules in subprograms external to modules to prevent compilation order requirements.

Module internal subroutines should primarily work on data declared within the module itself. If it requires data from another module, that data should come through the argument list. If so much data is required from other sources that the call list becomes unwieldy, the subprogram should be promoted to an external subprogram. 


\section{RELAP5-3D Developer Guidelines and Programming Practices}

\subsection{Executable Programming Rules}

Here are some rules for acceptable and unacceptable programming practices.

NO allocate/deallocate statements in the transient.

This has at least two negative effects on code performance. First, the unnecessary finding and setting aside of memory causes slowdowns. Second, it prevents shared-memory parallelism unless coupled by appropriate protection, namely, that only the first thread is allowed to allocate the memory. A subsequent attempt by another thread to allocate the same memory is an error.

Deallocate all allocated memory ASAP

RELAP5-3D can have multiple cases in a single input deck. Failure to deallocate can cause memory leaks and core dumps.

Initialize/Nullify pointers at creation

As soon as a pointer is created it should be initialized. Unless it is pointed to an existing value immediately upon creation, it should be nullified immediately.

Initialize variables at creation

Many global variables are created by modules. Most existing modules provide an "init" subroutine for initializing some or all of their variables. New modules should provide similar subroutines. Subroutine modmem also calls "init" subroutines from some modules.

In the case of other global variables, subroutine initdata provides the appropriate platform for initializing data.

Local variables should be initialized at the beginning of the subprogram. If the values must be saved from one call to the next, the initialization section should be protected by an if-test on a logical variable that is set to true before the subprogram is called and false inside the if-block. Note that this variable should be initialized in subroutine initdata so that it can be reset to true for each new case of an input deck. 


\section{RELAP5-3D Developer Guidelines and Programming Practices}

\subsection{RELAP5-3D Behavior}

This section covers code behavior such as file operations, stopping, input and output including code messages to users. Developers must ensure that the code continues to operate according to these principles. New coding, error fixes and maintenance and must produce the same code behavior.

RELAP5-3D code behavior is also known as the "Zen of RELAP." The ideal for RELAP5-3D performance is summarized as follows:

If the code gets through input processing, it should run the entire calculation.

This is an ideal for any computer code which few attain, particularly codes that are under continual development.

Another aspect of RELAP5-3D behavior: RELAP5-3D is polite. It does not destroy or overwrite certain important files, see Sec. 3.2. Instead it quits with an error message, see Sec 3.1.

Regarding input processing, Sec. 3.3, RELAP5-3D attempts to process the entire input file, even if it contains errors. It proceeds past input errors by loading default data so that it may continue to other parts of an input file and detect more errors. Getting as many error reports as possible is of great value to users, reducing correction time. Unfortunately, RELAP5-3D may have similar behavior to compilers, giving more error messages than existent errors because one error may generate several more, or the default data may not match well with other input.

During the transient, conditions may arise that cause the code to back up and possibly take a smaller time step or apply other adjustments. In some cases, it is important to inform the user and these are considered Warnings, Sec. 3.1. If the code cannot continue, it must give an error message, set the fail flag to terminate, and then leave the transient, see Sec 3.4. 


\section{RELAP5-3D Developer Guidelines and Programming Practices}

\subsection{Code Messages}

Error, warning, and informational messages produced by the code have a particular format associate with them that the users may key upon to quickly locate them in the printed output file. Both error and warning messages begin with a key field of 9 characters.

\subsubsection{Error Messages}

Formats that write an error message should begin with ' $0 * * * * * * * *$ ' (in other words, a zero followed by eight asterisks). This is important to those who teach RELAP5 training courses and for users who wish to quickly locate errors in their printed output files.

\subsubsection{Warning Message Formats}

Formats that write a warning message should begin with '0\$\$\$\$\$\$' (in other words, a zero followed by eight dollar signs). This is also important to those who teach RELAP training classes and for users who wish to quickly locate errors in their printed output files.

\subsubsection{Informational messages}

These messages are NOT preceded by a special field of 9 characters. They come out during input processing after the input deck echo and before the formal input edit. Such messages inform the user about progress in the processing of input, such as which record is being accessed from the restart and plot files, the format of the plot file, the solver selected by the user, etc. 


\section{RELAP5-3D Developer Guidelines and Programming Practices}

\subsection{File Operations}

When RELAP5-3D needs to either write information to or read information from a file, certain rules apply.

1. Important files may not be destroyed or overwritten by RELAP5-3D

a) These files include fluid property, printed output and restart

b) A special command line option allows the printed output file to be overwritten

2. If the user requests the overwriting of one of these important files, RELAP5-3D must quit with an error message. The error message should be written on the printed output file and on the screen output.

3. If specific content is required and not found, such as restart or plot information at a specific time or advancement number, an informational message must be written and the failure flag set so that the code quits gracefully (rather than with a core dump).

4. FILES SHOULD NOT BE OPENED OR CLOSED IN THE TRANSIENT. All opening of files should be done before the transient starts and all closes should take place after the transient.

It is very important for code performance to follow RULE 4. It takes time to open and close files and slows the code runtime unnecessarily. The slowdown gets worse if the open statement is inside a loop such as the time loop, and even worse if it is inside a deeper interior loop. 


\section{RELAP5-3D Developer Guidelines and Programming Practices}

\subsection{Input Processing}

The first goal of input processing is to process all of it without failing and to give the user as much feedback about input-file mistakes as possible. It is frustrating for a user to correct one or two reported input errors in their input file only to discover that there were more mistakes once the corrected file is processed.

The second goal of input processing is to place all the information into the correct modules and variables to run a transient or steady-state calculation correctly. This includes restart. The ideal towards which we strive is summarized as follows:

If the code gets through input processing, it should run the entire calculation.

Error messages should be as informative as possible, preferably stating which word of an input "card" is incorrect and what is wrong (data type, range, etc.), or how many words are required.

Input cards that are skipped because they make no sense, such as a 104 card on restart, should be flagged but not cause the code to fail. Missing required input cards and cards that are contradictory must cause failure. The code should set the failure flag, and write an error message, then warn the user that the code is proceeding with default data. For situations where appropriate default data cannot be ascertained, the code should warn the user about this, then fail immediately by calling the ABORT intrinsic.

Once the failure flag is set, it prevents future input cases from running. In an input "deck" with 18 cases, if there is a failure in input or transient processing in case 5 , then all successive cases fail in input processing. 


\section{RELAP5-3D Developer Guidelines and Programming Practices}

\subsection{Run Termination}

Besides input processing, there are situations in transient set-up and transient calculation that call for intentional failure. Some of these include:

1. Coefficient matrix is singular.

2. Failure flag is set to a terminal value.

3. A signal is sent by the PVM Executive to shut down.

4. File to read from or write on is closed.

5. A variable has a value in an impossible range, (causes division by zero, square root of a negative number, physically impossible, etc.)

6. An impossible state occurs, such as negative pressure, unstable fluid state, freezing coolant, melting temperature of solid walls.

There are two kinds of failures, graceful and immediate. The first three can fail gracefully while the latter three probably must fail immediately.

- Graceful Failure - Set the failure flag, write an error message, proceed directly to code error handling at the end of the current code section (input processing or transient loop). This allows final output dump to the printed output file and possible diagnostic dump (105 card).

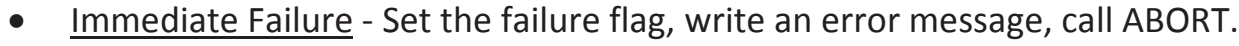

Calling the system routine ABORT triggers a core dump. 


\section{RELAP5-3D Developer Guidelines and Programming Practices}

\subsection{Output}

The code must inform the user about the results of its calculations. Efforts should be made to write output on existing files in appropriate places. The primary files for output are the printed output, restart, and plot files. Some special features have their own output files.

The printed output file organizes data into input summary, major and minor edits. Within each major section, there is a subdivision of information. When adding output to the printed output file, it must be done within the appropriate subsection of the appropriate section. For example new volume data must be reported in the volume subsection of both input processing and major edits, not in the heat structure section.

The restart file is organized according to modules. Each module has a restart read and restart write subroutine. These must match up. All the module's global data (not a local variable in a contained subprogram) should be written to and read from the restart file. 


\section{RELAP5-3D Developer Guidelines and Programming Practices}

\subsection{Performance and Testing}

RELAP5-3D must continue to adapt to new hardware, operating systems and compilers. Performance on these systems is to be maintained. The code must run fast and run correctly. Part of running fast is maintaining both vector and parallel performance.

\subsection{Vector Loop Processing}

Modern computer chips are designed with short vector loops that provide excellent code runtime reduction for loops that vectorize. Therefore, loops should be written to allow vector speed-up where possible. This is especially important in transient coding which is rerun with each call of its encompassing subprogram unit and may be executed hundreds, thousands, even millions of times.

Vector loops should have no recursion, subprogram calls, I/O, or inner loops. Even loops with an inner loop may vectorize on come platforms if the inner loop satisfies certain conditions. Also, calls to subprograms will not inhibit vectorization if they are intrinsic functions, statement functions, or can be internalized to the body of the loop through a compiler directive or option.

To develop vector loops, it is necessary to characterize them. The simplest characterization of a vector loop that has no calls or I/O and satisfies the following:

If the original loop were replaced by a collection of small loops, each having exactly one of its statements and the same do-loop index, start, end, and skip-factor, then the same final values would be calculated.

This is typically how vector calculations are performed. 


\section{RELAP5-3D Developer Guidelines and Programming Practices}

\subsection{Parallel Code}

Parallelism in RELAP5-3D is implemented with openMP paradigm. This paradigm applies to shared memory multi-core computer chips, which are found on most workstation and personal computer today.

Use of the openMP paradigm requires placement of openMP directives in the code to break out parallel sections and parallel loops. It also requires the use of a compiler option, generally given through a command line option, to activate it.

Shared memory parallelism is somewhat less restrictive than vectorization, but is nonetheless easy to break with recursion. It can also result in different calculations when the same loop is run several times due to the order of operations being done differently each time. Moreover it is very important not to parallelize any loop of section of code that is not inherently parallel, or the computer will generate incorrect results.

Preprocessors can detect parallelism inhibitors, but are not perfect and can indicate that a perfectly good loop should not be parallelized. It is safest to not force parallelization through use of directives that override the pre-compiler analysis.

(1) All Vector loops are parallel loops.

(2) I/O does not inhibit parallel.

(3) Calls to subprograms may or may not inhibit parallelism.

(4) Inner loops do not inhibit parallelism.

(5) Recursion inhibits parallelism.

(6) Parallelism can lead to different results on each run due to floating point round-off. 


\section{RELAP5-3D Developer Guidelines and Programming Practices}

\subsection{Code Testing}

The code must be well-tested to be robust. Testing reveals code errors introduced by code development, maintenance, and those introduced by fixing other errors. Developers must create tests, input files that exercise their coding, before their contributions to RELAP5-3D can be included in the main developmental line.

There are several levels of testing.

- New Feature Test Set

- Standard Test Set

- Developmental Test Set

- Specialty Test Set

When a code version is about to be released, either a subset or all of these tests are run. Any failed tests are recorded as User Problems (UP). If the code version is for external release, all the bugs are resolved before the code can be released.

\subsubsection{New Feature Testing}

Whenever a new feature is added, the developer must supply a set of input that test the feature. This set should include testing of input, including error messages for incorrect input, with the inp-chk option. It should also include test of some of the range of options for the input card words. At least one representative problem of each set will be incorporated in the standard installation test set described in Section 4.2.

\subsubsection{Standard Test Set}

These tests are run whenever an internal code version is released. There are several divisions of tests in the standard test set:

- Normal - Tests that are available for transmission to all users

- Athena - Tests that employ non-water fluids

- PVM - Problems that launch the PVM Executive to couple multiple RELAP5-3D runs to solve a single problem

- Other - Optional tests that do not go to all code users

Generally, when the code is released to the International RELAP5 Users Group (IRUG), the Normal and Athena problems are released. Clients who have the capability to use PVM now receive that test set. Changes to protected coding made in 2013 may allow the "Other" test set to be distributed to all IRUG members.

\subsubsection{Developmental Assessment}

These tests are summarized in Volume 3 of the RELAP5-3D Code Manuals. The tests provide developmental assessment cases that demonstrate and verify many of the models used in the code. This assessment uses a combination of phenomenological, separate effects, and integral effects cases to investigate how well selected code models perform. The code calculations are performed with both the semi- and nearly-implicit solutions schemes. 


\section{RELAP5-3D Developer Guidelines and Programming Practices}

The 17 phenomenological cases are simple problems, including thought problems with analytical solutions, that test one or two code models. The 27 separate effects cases are experiments that address one or a few code models. The 8 integral effects cases use data from large experiment facilities. These cases are generally of greater interest because they provide an indication of how well the code performs overall in modeling transients with a large number of phenomena. Eight specific tests were included in the assessment.

\subsubsection{Release Tests}

These test sets are also run when the code is released to the IRUG. These include:

- Dtstep Test Set that has approximately 2000 PVM-coupling problems

- TestDt a dozen problems of old issues with DTSTEP

- DOE test set

Other tests sets may be run also, depending on the client for whom the code is prepared. Also, if the code is configured for two or more client groups, each stripped version of the code is tested separately before release. 


\section{RELAP5-3D Developer Guidelines and Programming Practices}

\subsection{Living Document}

It is recognized that this is a "living" document and must be updated as languages, compilers, and computer hardware and software evolve. If you have questions, comments, or suggestions for improvements, please contact Dr. George Mesina at the Idaho National Laboratory. Your input will be evaluated and possibly included in future editions of the RELAP5-3D Developer Guidelines. 